\title{
LC-MS-Based Profiling Provides New Insights into Apocarotenoid Biosynthesis and Modifications in Citrus Fruits
}

\author{
Xiongjie Zheng ${ }^{\dagger}$, Jianing Mi ${ }^{\dagger}$, Xiuxin Deng ${ }^{\dagger}$, Salim Al-Babili ${ }^{\dagger}, *$ \\ ${ }^{\dagger}$ King Abdullah University of Science and Technology (KAUST), Division of \\ Biological and Environmental Science and Engineering, Center for Desert Agriculture, \\ the BioActives Lab, Thuwal 23955-6900, Saudi Arabia. \\ * Key Laboratory of Horticultural Plant Biology (Ministry of Education), Huazhong \\ Agricultural University, Wuhan 430070, China. \\ *Corresponding author: Salim Al-Babili (email: salim.babili@kaust.edu.sa)
}


A

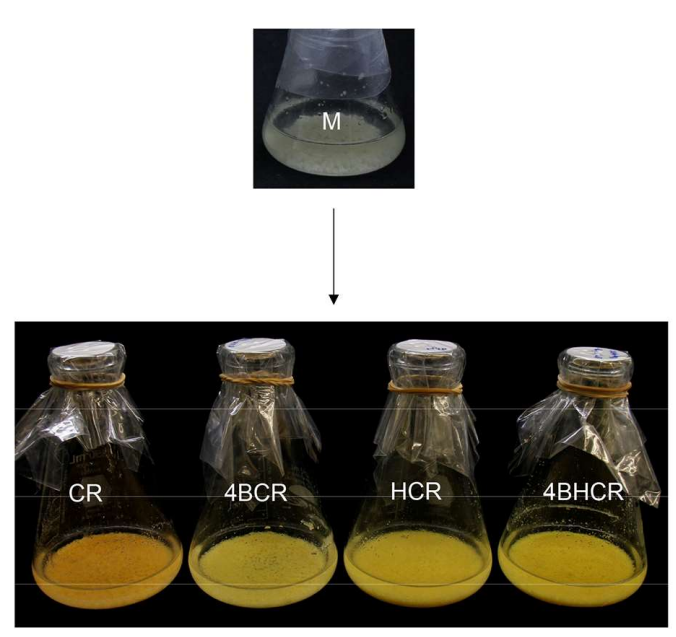

B

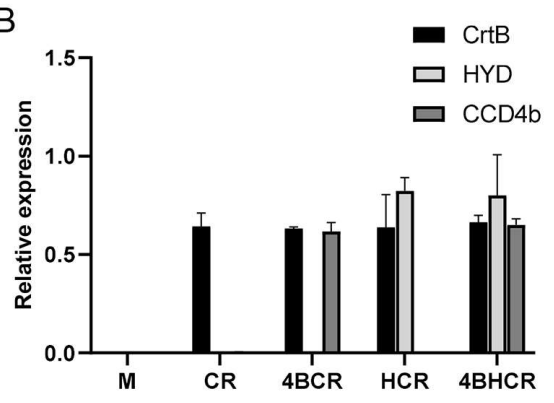

C

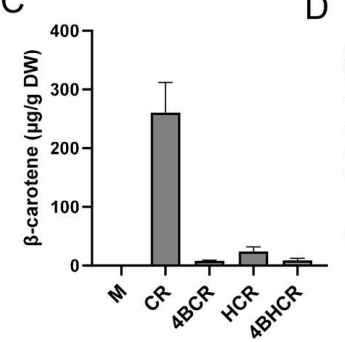

D

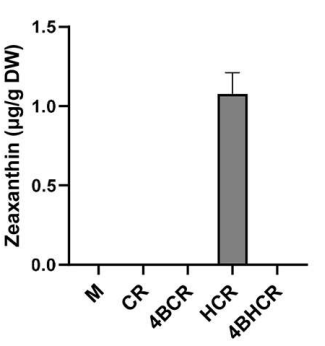

Figure S1. Expression levels of the transgenes and carotenoid content in transgenic citrus callus lines. (A) Suspension cultures of the M, CR, 4BCR, HCR and 4BHCR transgenic lines. (B) Expression levels of $C r t B, O s H Y D$ and $C i t C C D 4 b$ gene in these transgenic callus lines. ( $\mathrm{C}$ and $\mathrm{D})$ Content of $\beta$-carotene and zeaxanthin in these transgenic lines. 
A

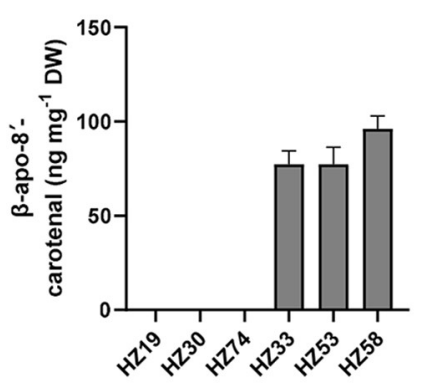

B

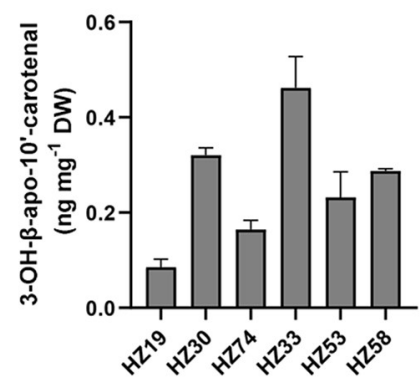

Figure S2. Levels of $\beta$-apo- 8 '-carotenal and 3-OH- $\beta$-apo-10'-carotenal among red or yellow peel of six citrus hybrids. 
Table S1. Gene-specific primers used in RT-qPCR analysis

\begin{tabular}{|c|c|c|}
\hline Gene name & Primer sequences $\left(5^{\prime} \rightarrow 3^{\prime}\right)$ & Reference \\
\hline \multirow[t]{2}{*}{$C r t B$} & Forward: TGGATTCCCAGTGAAGAAGGTC & Cao et al., $2012^{1}$ \\
\hline & Reverse: CATAATCTGCGCCATCATCAAG & \\
\hline \multirow[t]{2}{*}{ OsHYD } & Forward: ATCGCCAACGTGCCCTACT & This study \\
\hline & Reverse: GCACACCTTCGAACTTGTCCAT & \\
\hline \multirow[t]{2}{*}{ CitCCD $4 b$} & Forward: CAGCAAGAAATTTGGAGTTG & Rodrigo et al., $2013^{2}$ \\
\hline & Reverse: CGTAAAATCTTCTTGAGAC & \\
\hline \multirow[t]{2}{*}{ CitACTIN } & Forward: CCAAGCAGCATGAAGATCAA & Zheng et al., $2019^{3}$ \\
\hline & Reverse: ATCTGCTGGAAGGTGCTGAG & \\
\hline
\end{tabular}

(1) Cao, H.; Zhang, J.; Xu, J.; Ye, J.; Yun, Z.; Xu, Q.; Xu, J.; Deng, X. Comprehending crystalline $\beta$-carotene accumulation by comparing engineered cell models and the natural carotenoid-rich system of citrus. J. Exp. Bot. 2012, 63, 4403-4417.

(2) Rodrigo, M. J.; Alquezar, B.; Alos, E.; Medina, V.; Carmona, L.; Bruno, M., et al., A novel carotenoid cleavage activity involved in the biosynthesis of Citrus fruit-specific apocarotenoid pigments. J. Exp. Bot. 2013, 64, 4461-78.

(3) Zheng, X.; Zhu, K.; Sun, Q.; Zhang, W.; Wang, X.; Cao, H., et al., Natural Variation in CCD4 Promoter Underpins Species-specific Evolution of Red Coloration in Citrus Peel. Mol. Plant. 2019, 12, 1294-1307. 